\title{
Spatial-frequency uncertainty and cuing effects on psychometric functions for contrast detection
}

\author{
YOSHIO OHTANI \\ Kyoto Institute of Technology, Kyoto, Japan \\ and \\ SHUJI MORI \\ Tokyo Metropolitan University, Tokyo, Japan
}

\begin{abstract}
In the present study, the effects of spatial-frequencyuncertainty and cuing on psychometric functions for contrast detection of sinusoidal gratings are examined. For this purpose, psychometric functions were collected from 4 subjects under fixed-frequency, randomized-frequency, and cued-frequency conditions. The experiment was conducted with a temporal two-alternative forced-choice task, and five spatial frequencies in the range of 0.5 and $8.0 \mathrm{c} / \mathrm{deg}$ and seven contrast levels for each frequency were used. The results showed that the psychometric functions for the randomized-frequency condition were shallower than those for the fixed-frequency condition, supporting the single-band model for the uncertainty effects (Hübner, 1993a, 1993b). For the cued-frequency condition, the slopes of the functions were not clearly different from those for the randomized condition. These results clearly differ from those of Hübner (1996b), which showed, in the spatial two-alternative forced-choice task, steeper psychometric functions for the randomized-frequency condition than those for the fixed-and cued-frequency conditions, supporting the multiple-band model (Hübner, 1993a, 1993b). The difference suggests that the single-band model applies to the uncertainty effects in the temporal forced-choice task, whereas the multiple-band model does so in the spatial forced-choice task.
\end{abstract}

Human observers perceive a spatial pattern less efficiently when they do not know in advance what to perceive than when they do. In visual psychophysics, such phenomena are known as uncertainty effects and are studied with respect to spatial frequency for sinusoidally modulated black/white patterns, or gratings, as stimuli. Experimentally, uncertainty is introduced by varying the frequencies of grating stimuli randomly across trials so that the observers are uncertain about the stimulus frequency to be presented on each trial. Research has shown that the observer's performance for detecting, discriminating, and identifying grating stimuli is lowered under the frequency uncertainty situations, as compared with no-uncertainty situations in which the spatial frequency is fixed throughout trials (e.g., Kramer, Graham, \& Yager, 1985; Yager, Kramer, Shaw, \& Graham, 1984). It

The experiment, conducted at Toyama Prefectural University, was part of the graduation thesis of Masayuki Mizuno. The data were previously reported in a somewhat different form at the winter meeting of the Vision Society of Japan, Tokyo, January 1998, and at the annual meeting of the International Society for Psychophysics, Québec, August 1998. The study was partly supported by Grants 09710054 and 11610083 from the Japanese Ministry of Education, Science and Culture to S.M. We thank Masayuki Mizuno and Sadanori Oka for data collection and Yoshimichi Ejima for helpful comments throughout the research. Correspondence concerning this article should be addressed to Y. Ohtani, Faculty of Engineering and Design, Kyoto Institute of Technology, Matsugasaki, Sakyo-ku, Kyoto 606-8585, Japan (e-mail: ohtani@hiei.kit.ac.jp). is also known that such frequency uncertainty effects can be compensated for by providing the observer with information, or a cue, about the spatial frequency of the signal to be presented. Presenting cues (identical or similar to the signals) shortly before the signal improves performance in randomized-frequency situations, sometimes up to the level for fixed-frequency situations (Davis, Kramer, \& Graham, 1983; Hübner, 1996a, 1996b).

Theoretical explanations for the spatial-frequency uncertainty effects are usually classified into two types, single-band and multiple-band models (for a review, see Graham, 1989; Hübner, 1993b). The two models are built on a general framework of the multiple-channel mechanism of human vision. In this framework, the human visual system consists of multiple channels, each of which is tuned to a restricted range or band of spatial frequency (Blakemore \& Campbell, 1969; Campbell \& Robson, 1968; also see Wilson, 1991, for reference). Single-band models assume that the observers monitor only one channel (or a subset of channels) at a time and, in uncertainfrequency situations, switch their monitoring to different channels from trial to trial. Such trial-by-trial switching has two effects. One is that the observers sometimes monitor an inappropriate channel that is not stimulated by a signal on that trial (Davis \& Graham, 1981; Davis et al., 1983). The other is that the observers are likely to monitor a somewhat mistuned channel for the signal, because they are unable to place their monitoring at the optimal frequency channel for the signal after switching from 
the other channel on the previous trial (Hübner, 1993a, 1993b). In either case, a decrement in signal detectability is expected.

Multiple-band models assume that when observers are uncertain about the signal frequency, they monitor several channels simultaneously in order to "hit" the signal. Since the observers base their decision on the outputs of multiple channels, all but one of which are not stimulated by the signal on a given trial, the decision will inevitably be affected by internal noise of the nonstimulated channels (Creelman, 1960; Pelli, 1985). This leads to a decrement in detection performance, particularly when the signal intensity is so low that the output of the stimulated channel is not very different from the outputs of the nonstimulated channels (see below).

An important question is which of the two models is more appropriate for explaining empirical results. One useful approach is to measure psychometric functions in a two-alternative forced-choice (2AFC) detection task with and without frequency uncertainty (Hübner, 1996b). In the 2AFC task, a grating stimulus is presented in one of two spatially or temporally separated intervals in a trial, and the observer has to decide which of the two intervals contains the stimulus. The psychometric function is a plot of proportion of correct detection, $P C$, as a function of the stimulus contrast (see the Method section). The contrast level corresponding to $75 \% P C$ in the function is usually taken as the contrast threshold. As the signal detectability is decreased owing to the uncertainty effects, the psychometric function moves to the right along the contrast axis (which corresponds to the increase in threshold). Computational analysis of single-band and multiple-band models shows differential changes of the slope of psychometric functions when the functions move to the right with increasing uncertainty: shallower slopes for the single-band models and steeper slopes for the multiple-band models (Hübner, 1993a). Figure 1 illustrates the predicted changes of psychometric functions.

For both models, the predictions are based on idealobserver analysis of the 2AFC task (Green \& Swets, 1966; Pelli, 1985). According to the analysis, the observers in no-uncertainty situations make their decision by comparing two outputs, or samples, provided by the monitored channel, one for the interval containing the signal and the other for the "blank" interval. The decision is correct if the sample for the signal interval is larger than that for the blank interval. Representing the two samples as normal random variables, one can compute $P C$ as a normal distribution function of the stimulus contrast (indicated by solid curves in both panels of Figure 1). In cases of frequency uncertainty, the predictions for the single-band model have been derived by Hübner (1993a) with an assumption that the monitored channel is mistuned, the magnitude of which varies randomly across trials. This increases the variance of channel outputs (samples) across trials, as compared with optimal tuning in no-uncertainty situations. Moreover, the

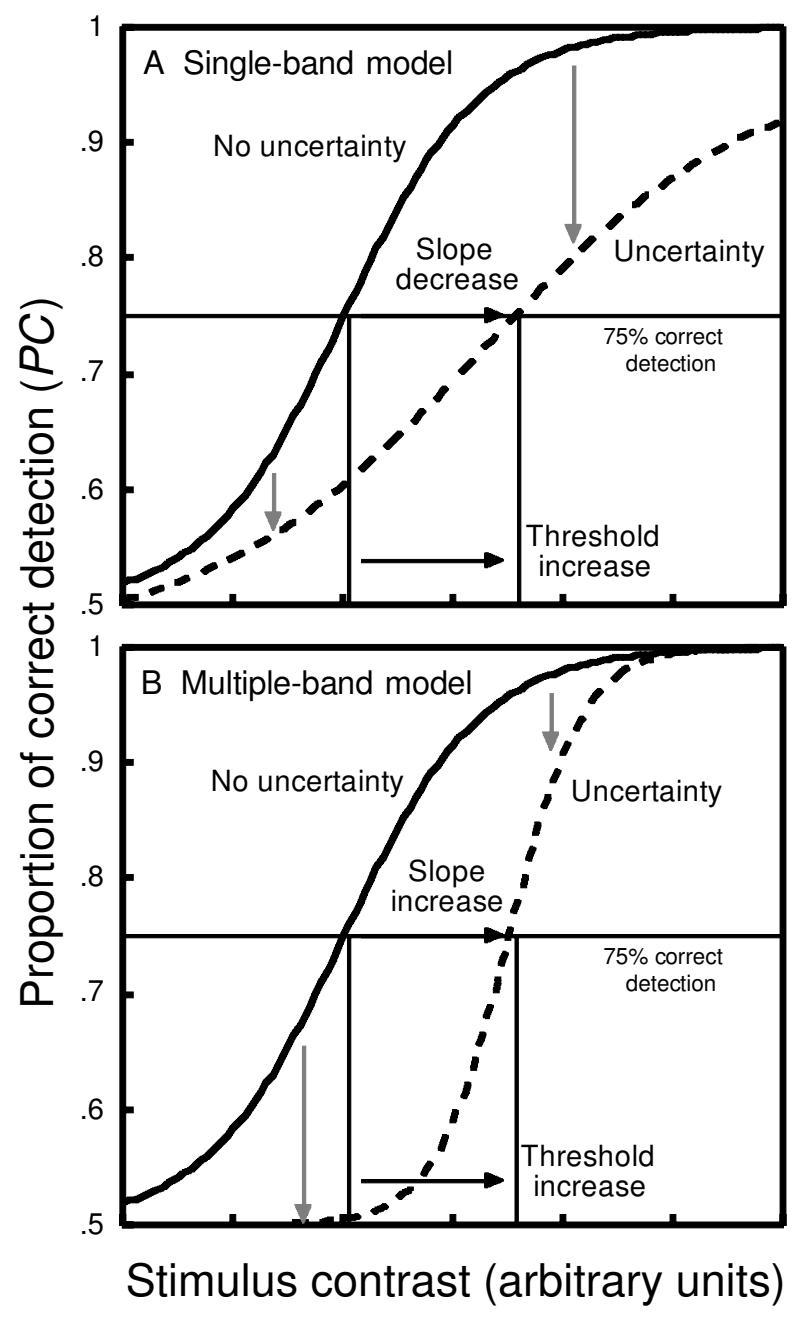

Figure 1. Schematic illustration of the predictions for the singleband (A) and the multiple-band (B) models. See the text for details.

mistuning reduces the size of output for the signal interval, because it makes the channel less effective in processing the signal. Incorporating these changes from channel mistuning into the ideal-observer analysis, Hübner (1993a) has shown the predicted change of the psychometric functions; as is readily seen in Figure 1A, the function (indicated by a broken curve) is flattened, with a higher threshold contrast under frequency uncertainty. The shallow slope mainly reflects the larger variance of the underlying normal probability function of the channel output, and the higher threshold corresponds to the low detectability that is due to the smaller output for the signal interval. ${ }^{1}$

Whereas the single-band model incorporates nonoptimal properties of the monitored channel into the idealobserver analysis, the multiple-band model is concerned with the decision rule of the analysis in order to combine the outputs of multiple channels into a single decision 
variable. It is assumed that the observers choose the interval that contains the maximum output among the monitored channels (Creelman, 1960; Pelli, 1985). The decision is correct if the maximum output is from the stimulated channel- that is, the output of that channel is larger than any of the outputs of the nonstimulated channels. Representing the outputs of the channels, stimulated or nonstimulated, as random variables across trials, owing to internal noise in each channel, one can compute theoretical psychometric functions as shown in Figure $1 \mathrm{~B}$. In the panel, the effect of an increasing number of monitored channels with increasing uncertainty (indicated by a broken curve) is larger for low stimulus contrast (at the lower part of the function) than for high contrast. Note that the output of the stimulated channel is a function of the stimulus contrast. For low contrast, the output of the stimulated channel will be so small that, under uncertainty situations, one (or more) of the outputs of the nonstimulated channels, perturbed by internal noise, may exceed the output of the stimulated channel. This results in a large reduction in $P C$. On the other hand, $P C$ for high contrast is relatively unaffected, because the output of the stimulated channel will be much larger than the output of the nonstimulated channels. This uneven effect of increasing the number of channels on $P C$ steepens the psychometric functions when they move to the right.

Up to now, the only empirical test of these predictions was provided by Hübner (1996b). For contrast detection of sinusoidal gratings with a spatial 2AFC task, Hübner (1996b) employed a randomized-frequency condition and several cued-frequency conditions, and comparing the resulting psychometric functions with those obtained for a fixed-frequency condition, he found that, for the randomized-frequency condition, the psychometric functions shifted to the right and steepened, as compared with those for the fixed-frequency condition. For one of the cued-frequency conditions in which the cues were gratings with the same frequency as the signals, the psychometric functions were indistinguishable from those obtained for the fixed-frequency condition, indicating that the cues compensated for frequency uncertainty almost entirely (also see Hübner, 1996a).

The steepening of the psychometric functions for the randomized-frequency condition, as compared with those for the fixed-frequency condition, led Hübner (1996b) to conclude that the multiple-band model would be more appropriate than the single-band model for explaining the uncertainty effects. Before accepting this conclusion as a general rule for frequency uncertainty effects, two points should be noted about the results of his experiment. First, the threshold contrasts obtained for the fixedfrequency condition were rather high (e.g., 0.0669 at $4.14 \mathrm{c} / \mathrm{deg}$ ), and they showed a monotonically increasing characteristic with respect to spatial frequency. This was probably due to a background noise pattern (i.e., a grating whose component stripes vary in width randomly across space) added to the signal gratings, as Hübner (1996b) noted, and a relatively short signal duration, 106 msec, in his experiment (see Arend, 1976; Schober \& Hilz, 1965). The threshold contrasts obtained for a longer duration without external noise are usually much lower (typically less than 0.01 at around $4 \mathrm{c} / \mathrm{deg}$ ) and show a Ushaped characteristic (e.g., Campbell \& Robson, 1968). Second, the size of uncertainty effects varied with the signal frequency. For the randomized-frequency condition, the contrast thresholds for all the frequencies were higher than those for the fixed-frequency condition, but the difference between the two conditions decreased as the frequency increased. Such frequency-dependent uncertainty effects have not been reported in previous studies (e.g., Kramer et al., 1985; Yager et al., 1984).

These two results circumscribe the generality of Hübner's (1996b) findings and conclusions for the frequency uncertainty effects. Given the significance of the framework presented by his work (Hübner, 1993a, 1996b) and the scarcity of the empirical data, we decided to reexamine and extend his empirical findings in our own study. For this purpose, the present study differed from Hübner's (1996b) study in some respects: The stimuli were presented with no external noise and for a relatively long duration $(500 \mathrm{msec})$, and a temporal version of the $2 \mathrm{AFC}$ task was used (see below). These methodologies have been frequently used (at least as frequently as those of Hübner's, 1996b, study) in studies of contrast detection for grating stimuli. In addition, the present study used cues similar to Hübner's (1996b), but at a much lower contrast level with longer duration and postcue interval.

\section{METHOD}

\section{Subjects}

Four male subjects with normal or corrected-to-normal visual acuity participated in this experiment (ages between 21 and 37 years). One of them (S.M.) was the second author, and the others (S.O., M.M., and A.O.) were either graduate or undergraduate students. S.O. and M.M. were well aware of the purpose of the present study, whereas A.O. was naive as to it.

\section{Apparatus and Stimuli}

The stimuli were presented on a 17-in. color CRT monitor that was driven by a stimulus generator (Neuroscientific Inc., Venus model 1020) with a $90-\mathrm{Hz}$ frame rate and a 12-bit (gamma-corrected) luminance resolution for each of the RGB channels. The stimulus presentation, timing control, and response collection were controlled by a personal computer.

The stimuli were horizontally oriented sinusoidal gratings presented inside a circular window of $5^{\circ}$ diameter on a dark background. The mean luminance of the stimuli was approximately $65 \mathrm{~cd} / \mathrm{m}^{2}$. Except for the stimulus presentation, the stimulus window was kept homogeneous at the mean luminance level throughout the experimental session. Five different spatial frequencies were used for the grating stimuli: $0.5,1.0,2.0,4.0$, and $8.0 \mathrm{c} / \mathrm{deg}$. The stimuli were viewed monocularly through an aperture of $1-\mathrm{cm} \mathrm{di-}$ ameter, which was placed just in front of one eye of the subject.

Before the main experiment, the threshold contrast (75\% correct level) of each spatial frequency was estimated for each subject by fitting a logistic function (see Equation 2 below) to the data sets 
collected in a preliminary experiment. The contrast levels were measured in Michelson contrast $\left(L_{\max }-L_{\min }\right) /\left(L_{\max }+L_{\min }\right)$. The estimated threshold contrast $\left(x_{0}\right)$ was then used as the criterion sensation level (0 SL), where $S L$ is defined as:

$$
S L=20 \times \log \left(x / x_{0}\right) .
$$

In the main experiment, seven contrast levels $(x)$ in the range of $-6 S L$ to $6 S L$ were employed for each spatial frequency.

The cue was a grating stimulus identical to the signal to be presented on that trial, except that its contrast level was three times higher than the threshold contrast $\left(x_{0}\right)$ for each spatial frequency and for each subject. The levels varied across subjects and frequencies but did not exceed 0.5 (in Michelson contrast; cf. Hübner, 1996b).

\section{Procedure}

Before each experimental session, the subjects were dark adapted for $3 \mathrm{~min}$ and then adapted to the mean luminance of the signal for $3 \mathrm{~min}$. The experiment was conducted with a temporal 2AFC task. For each trial, there were two stimulus intervals of $500 \mathrm{msec}$ each, separated by $1,300 \mathrm{msec}$, and the signal was presented in one of the two intervals with equal probability. The stimulus intervals were indicated by buzzer sounds. The subject responded by pressing one of two keys to indicate which interval contained the stimulus. No feedback was given for the response.

There were three conditions in this experiment—namely, fixed frequency, randomized frequency, and cued frequency. In the fixedfrequency condition, the spatial frequency of the signal was kept constant throughout a block of 175 trials. In the randomized-frequency condition, the spatial frequency was varied randomly (from a set of five frequencies) across trials within a block. The five frequencies were chosen equally often in the block. In the cued-frequency condition, the spatial frequency was varied across trials, as in the randomized condition, but a cue was presented on each trial before the first stimulus interval. The duration of the cue was $500 \mathrm{msec}$, and the postcue interval before the first stimulus interval was $1,300 \mathrm{msec}$. The cue presentation was indicated by a buzzer sound.

For each subject, data were collected from 20 blocks of 175 trials for each of the three conditions (a total of 60 blocks). There were seven contrast levels for each of the five frequencies in each condition, and the subject made a total of 100 responses per contrast level for each frequency. The blocks of different conditions were intermixed for each subject in order to counterbalance the possible day-by-day variation of the subject's performance. Before data collection, all the subjects participated in a preliminary experiment that consisted of 20-60 blocks (a total of 3,500-10,500 trials) for the three conditions.

\section{RESULTS}

For each subject's data, psychometric functions were obtained by plotting $P C$ as a function of $S L$ separately for each condition and frequency. Each of the individual psychometric functions was fitted by a logistic function

$$
P C(S L)=0.5+0.5 /\{1+\exp [-\alpha(S L-\beta)]\},
$$

where the parameters $\alpha$ and $\beta$ correspond to the slope and the threshold sensation level, respectively, at $75 \%$ $P C .^{2}$ The best-fitting functions were obtained by the least-squares method.

Figure 2 presents psychometric functions for the average data of the 4 subjects, together with logistic functions fitted to the average data. Although the slopes and thresholds of the average data are not the same as the mean slopes and thresholds of the 4 subjects (see Tables 1 and 2 below), the average data are representative of an overall pattern of the individual data. It can be readily seen in the figure that the psychometric functions for the three conditions are positioned close to each other (cf. Hübner, 1996b), but there are noticeable differences among them. For the fixed-frequency condition, the functions are steeper than those for the randomized-frequency and for the cued-frequency conditions. For the randomizedfrequency condition, the functions are slightly shifted to the right of those for the fixed-frequency condition. For the cued-frequency condition, the functions are shifted to the left of those for the other two conditions, and their slopes are similar to those for the randomized condition but shallower than those for the fixed condition.

These observations are in general agreement with the pattern observed for the individual subjects and are supported by statistical analyses using the slope and threshold parameter values ( $\alpha$ and $\beta$ ) estimated from the individual data. Tables 1 and 2 show the slope and threshold parameter values, respectively, for the 4 subjects. In a comparison between the fixed and the randomized conditions, Wilcoxon matched-pair signed-rank tests with all 20 pairs (4 subjects $\times 5$ frequencies) showed that the slopes were significantly smaller $(T=23, p<.01)$ and the thresholds were significantly higher $(T=31, p<.01)$ for the randomized condition than for the fixed condition. In a comparison between the randomized and the cued conditions, a Wilcoxon test for the slopes showed no significant difference $(T=76, p>.2)$, but the thresholds were higher for the randomized condition for all 20 pairs. In a comparison between the fixed and the cued conditions, Wilcoxon tests showed significantly larger slopes $(T=33, p<.01)$ and higher thresholds $(T=28$, $p<.01)$ for the fixed condition.

The average data (Figure 1), as well as the individual data (Tables 1 and 2), showed that the differences that were due to the condition varied with signal frequency, but not in an orderly way. Repeated measures two-way analyses of variance showed a significant main effect of condition for the threshold values $(p<.01)$ and marginally significant main effects of condition and frequency for the slopes $(p s<.1)$, but their interaction was not significant for either slope or threshold values. The small number of data precludes any general conclusion about the interaction.

Figure 3 presents the mean contrast thresholds for the fixed-frequency condition (converted from the sensation levels reported in Table 2) as a function of spatial frequency. For comparison, the contrast thresholds obtained in Hübner's (1996b) blocked (fixed-frequency) condition are plotted together. As can be readily seen, the thresholds of the present experiment are much lower than those of Hübner (1996b), and they show a U-shaped characteristic typically observed for contrast detection of sinusoidal gratings (Wilson, 1991). Similar patterns 

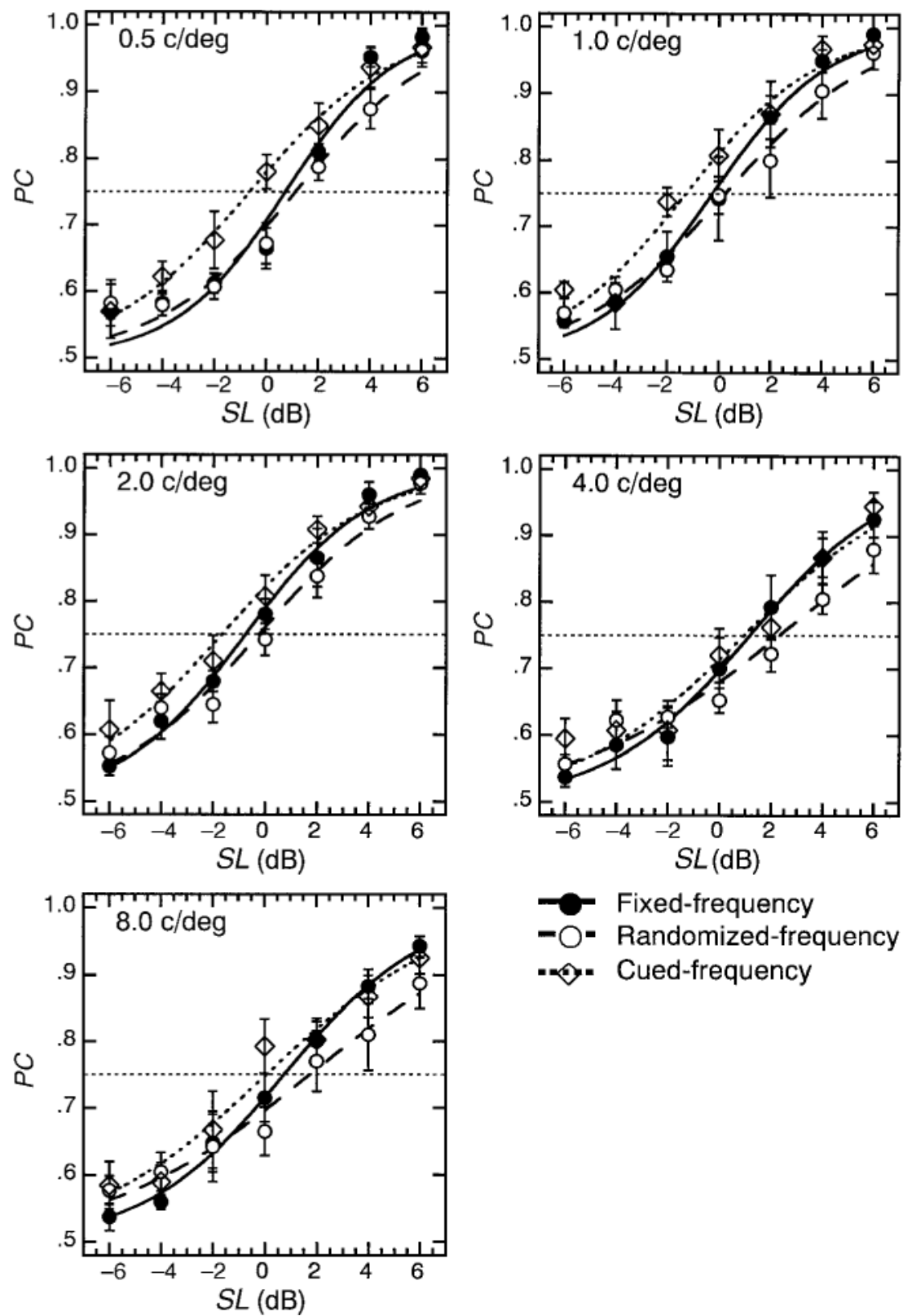

Figure 2. Average psychometric functions, proportion of correct detection $(P C)$ against sensation level $(S L)$, for the 4 subjects in the three conditions and the five signal frequencies. Curves are best-fitting logistic functions for each set of the average data, and vertical bars indicate the standard errors of the mean.

were also observed for the randomized-frequency and cued-frequency conditions (data not shown here).

\section{DISCUSSION}

The present experiment demonstrated the effects of spatial-frequency uncertainty and cuing on psychometric functions (Figure 2), although the size of the effects was relatively small (cf. Hübner, 1996b). In addition, the contrast thresholds showed a U-shaped characteristic with respect to the spatial frequency (Figure 3), unlike the monotonically increasing pattern observed in Hübner (1996b). The difference in contrast threshold was probably due to the relatively long signal duration and absence of external noise in the present experiment.

The present finding of shallower slopes for the randomized-frequency condition, as compared with those for the fixed-frequency condition, is consistent with the 
Table 1

Estimated Slope Parameter $\alpha$ of Psychometric Functions for Each Subject

\begin{tabular}{clccccc}
\hline & & \multicolumn{4}{c}{ Spatial Frequency $(\mathrm{c} / \mathrm{deg})$} \\
\cline { 3 - 6 } Subject & Condition & 0.5 & 1.0 & 2.0 & 4.0 & 8.0 \\
\hline S.M. & Fixed & 0.43299 & 0.55181 & 0.43792 & 0.59297 & 0.54819 \\
& Randomized & 0.53604 & 0.38211 & 0.53728 & 0.25493 & 0.55755 \\
& Cued & 0.48382 & 0.37288 & 0.46066 & 0.55208 & 0.45204 \\
S.O. & Fixed & 0.37325 & 0.54386 & 0.42177 & 0.34191 & 0.37522 \\
& Randomized & 0.37164 & 0.42888 & 0.34114 & 0.36596 & 0.24700 \\
& Cued & 0.49640 & 0.54314 & 0.36318 & 0.34780 & 0.35321 \\
M.M. & Fixed & 0.82507 & 0.52184 & 0.64838 & 0.38659 & 0.30870 \\
& Randomized & 0.27475 & 0.26867 & 0.35784 & 0.20106 & 0.23861 \\
& Cued & 0.20240 & 0.39105 & 0.28593 & 0.19450 & 0.19609 \\
A.O. & Fixed & 0.49209 & 0.46076 & 0.31069 & 0.33245 & 0.37872 \\
& Randomized & 0.37925 & 0.38752 & 0.30013 & 0.19887 & 0.15914 \\
& Cued & 0.39843 & 0.36414 & 0.34085 & 0.28098 & 0.29253 \\
Meanyyyyy & Fixed & 0.53085 & 0.51957 & 0.45469 & 0.41348 & 0.40271 \\
& Randomized & 0.39042 & 0.36680 & 0.38410 & 0.25521 & 0.30058 \\
& Cued & 0.39526 & 0.41780 & 0.36266 & 0.34384 & 0.32347 \\
\hline
\end{tabular}

single-band model for the uncertainty effects (Hübner, 1993a). This is in contrast to Hübner's (1996b) finding of steeper slopes with increasing uncertainty, supporting the multiple-band model. This difference may be attributed to the experimental tasks and/or to the presence or absence of the background noise pattern. The present experiment was conducted with a temporal $2 \mathrm{AFC}$ task, whereas Hübner (1996b) used a spatial 2AFC task, where the signal was presented in one of two spatially separated fields. In the spatial 2AFC task, the observers must distribute their attention spatially over the two stimulus fields in order to detect the signal. Furthermore, their spatial attention could have been distracted by the highcontrast background noise pattern used in Hübner's (1996b) study. A recent model by Kontsevich and Tyler (1999) has shown that such distraction of attention will change the shape of the psychometric function in a manner similar to the spatial-frequency uncertainty in the multiple-band model with a maximum-output rule (e.g.,
Pelli, 1985). The threshold and slope of the psychometric function will increase as the size of distraction increases, even when the observers are certain about the location and timing of the stimulus presentation. It is thus likely that in Hübner's (1996b) study, the psychometric functions were affected not only by the spatial-frequency uncertainty, but also by distraction of spatial attention. In the present study, the signal was presented in the same spatial field with no external noise on each trial so that the observers might be able to focus their attention properly on the stimulus field. This suggests that the operation of spatial attention may have contributed to the discrepant findings of the shape of psychometric functions between the two studies. The difference in the operation of spatial attention also accounts for the relatively small changes in the psychometric functions observed in the present study. Future studies will need to clarify how much contribution spatial operation makes to changing psychometric functions, in different tasks with and without external noise,

Table 2

Estimated Threshold Parameter $\beta$ of Psychometric Functions for Each Subject

\begin{tabular}{llccccc}
\hline & & \multicolumn{5}{c}{ Spatial Frequency $(\mathrm{c} / \mathrm{deg})$} \\
\cline { 3 - 6 } Subject & Condition & 0.5 & 1.0 & 2.0 & 4.0 & 8.0 \\
\hline S.M. & Fixed & -0.044555 & 1.4768 & -1.4073 & 0.96805 & 1.0815 \\
& Randomized & 1.4452 & 1.4522 & 0.56104 & 2.5171 & 2.0746 \\
& Cued & -0.56662 & 0.36997 & 0.23742 & 2.1637 & 1.0367 \\
S.O. & Fixed & 0.93004 & -1.5905 & -1.0562 & -1.6125 & -0.70891 \\
& Randomized & 1.4617 & -1.1021 & -1.3797 & 1.0449 & 0.15205 \\
& Cued & 0.51640 & -2.2682 & -2.3669 & -1.5211 & -2.2506 \\
M.M. & Fixed & 1.4950 & 0.62723 & -0.62336 & 2.0205 & 0.14340 \\
& Randomized & 2.0169 & 1.7995 & -0.72021 & 3.2065 & -0.48125 \\
& Cued & -0.11918 & -0.15136 & -2.9482 & 1.6066 & -1.3239 \\
A.O. & Fixed & 0.40846 & -1.2692 & 0.27981 & 3.2991 & 2.4143 \\
& Randomized & -0.40667 & -0.50881 & 0.55243 & 3.2862 & 6.8090 \\
& Cued & -2.3302 & -2.7606 & -1.9485 & 1.3592 & 2.3949 \\
Mean & Fixed & 0.69724 & -0.18892 & -0.70176 & 1.1688 & 0.73257 \\
& Randomized & 1.1293 & 0.41020 & -0.24661 & 2.5137 & 2.1386 \\
& Cued & -0.62490 & -1.2025 & -1.7565 & 0.90210 & -0.035725 \\
\hline
\end{tabular}




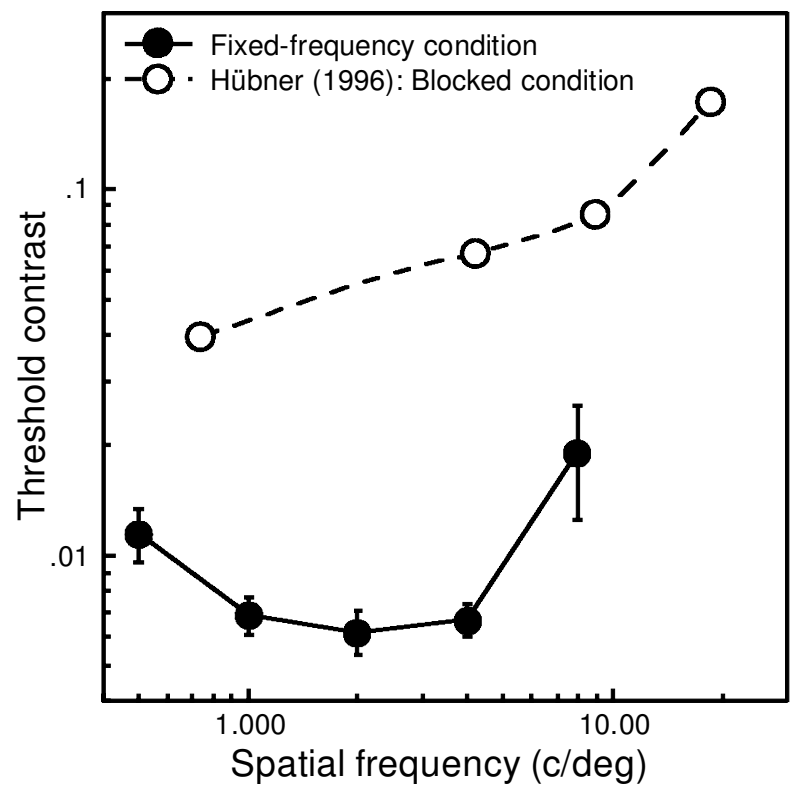

Figure 3. Mean estimates of contrast thresholds (at $75 \%$ of correct detection) as a function of spatial frequency for the fixedfrequency condition of the present experiment and the blocked condition of Hübner (1996b). Vertical bars indicate the standard errors of the mean.

in order to understand the underlying mechanism of the frequency uncertainty effects (i.e., single-band and multiple-band models).

The results of the cued-frequency condition do not support either the single-band or the multiple-band model outlined in the introduction. Although the thresholds were significantly lower for the cued-frequency than for the randomized-frequency condition, indicating that presenting cues reduced the frequency uncertainty, the slopes were not significantly different between the two conditions. A close inspection of the individual data (Table 1) reveals that the insignificant difference in slope resulted from the opposing tendencies among the subjects, rather than from the within-subjects invariance; 2 subjects (S.O. and A.O.) showed steeper slopes for the cued condition for four out of five spatial frequencies, whereas the other 2 (S.M. and M.M.) showed just the opposite. Thus, the results of the former 2 subjects are consistent with the single-band model, whereas those of the latter two are consistent with the multiple-band model. Sizable individual differences in detection performance have been reported under frequency uncertainty situations (Davis \& Graham, 1981; Davis et al., 1983), but no mechanism or factor responsible for the individual differences has been identified.

It should be also noted that, for all the subjects, the thresholds for the cued-frequency condition were consistently lower than those for the fixed-frequency condition. The previous studies have shown that presenting cues improves the detectability, sometimes up to the level for fixed-frequency situations (Davis et al., 1983;
Hübner, 1996a, 1996b), but no study has reported lower thresholds for the cued-frequency condition, as compared with the fixed-frequency condition. We can think of a few possibilities, although none of them seems to be very likely. One possibility is that, in the cued-frequency condition, the cue signal might enhance the sensitivity of that channel, leading to an improved detectability for the following signal. Another possibility is that, in the fixed condition, the signal on the previous trial might have caused forward masking and reduced the detectability for the signal on the next trial. We think these possibilities unlikely because, in the present experiment, the postcue interval $(1,300 \mathrm{msec})$ and the intertrial interval $(>2,000 \mathrm{msec})$ were rather long and the contrasts of the cues and the signals were no more than three times higher than the threshold. The other possibility is that, because the main experiment took a long time (a total of 60 sessions), the day-by-day variability of the subjects' performance happened to cause the relatively low thresholds for the cued condition. However, our subjects participated in a preliminary experiment of 20-60 blocks, and their performance appeared to be asymptotic for all the conditions before the main experiment. Altogether, further elaboration of the single-band and the multipleband models is required to account for the present results of cuing effects on the thresholds and the slopes of psychometric functions.

In summary, the present experiment showed that the spatial-frequency uncertainty and cues affected the psychometric functions for the temporal $2 \mathrm{AFC}$ contrast detection task with stimuli of relatively long duration and no external noise. The results suggest that, in the present experimental conditions, the single-band model would be appropriate for explaining the uncertainty effects obtained for the randomized-frequency condition (in comparison with the fixed-frequency condition). The differences between the present results and those of Hübner (1996b) could be attributed to the tasks and the stimulus parameters used in the two studies.

\section{REFERENCES}

Arend, L. E. (1976). Response of the human eye to spatially sinusoidal gratings at various exposure durations. Vision Research, 16, 1311-1315.

Blakemore, C., \& Campbell, F. W. (1969). On the existence of neurons in the human visual system selectively sensitive to the orientation and size of retinal images. Journal of Physiology, 203, 237-260.

CAmpbell, F. W., \& Robson, J. G. (1968). Application of Fourier analysis to the visibility of gratings. Journal of Physiology, 197, 551-566.

Creelman, C. D. (1960). Detection of signals of uncertain frequency. Journal of the Acoustical Society of America, 32, 805-810.

DAVIS, E. T., \& GRAHAM, N. (1981). Spatial frequency uncertainty effects in the detection of sinusoidal gratings. Vision Research, 21, 705-712.

Davis, E. T., Kramer, P., \& Graham, N. (1983). Uncertainty about spatial frequency, spatial position, or contrast of visual patterns. Perception \& Psychophysics, 33, 20-28.

GraHAM, N. (1989). Visual pattern analyzers. New York: Oxford University Press.

Green, D. M., \& Swets, J. A. (1966). Signal detection theory and psychophysics. New York: Wiley.

HüBNER, R (1993a). Different ways of modeling spatial-frequency uncertainty in visual signal detection. Biological Cybernetics, 69, 457462 . 
HÜBNer, R. (1993b). On possible models of attention in signal detection. Journal of Mathematical Psychology, 37, 266-281.

HÜBNER, R. (1996a). The efficiency of different cue types for reducing spatial-frequency uncertainty. Vision Research, 36, 401-408.

HÜBNER, R. (1996b). Specific effects of spatial-frequency uncertainty and different cue types on contrast detection: Data and models. Vision Research, 36, 3429-3439.

Kontsevich, L. L., \& Ty Ler, C. W. (1999). Distraction of attention and the slope of the psychometric function. Journal of the Optical Society of America A, 16, 217-222.

Kramer, P., Graham, N., \& Yager, D. (1985). Simultaneous measurement of spatial-frequency summation and uncertainty effects. Journal of the Optical Society of America A, 2, 1533-1542.

Pelli, D. G. (1985). Uncertainty explains many aspects of visual contrast detection and discrimination. Journal of the Optical Society of America A, 2, 1508-1532.

Schober, H. A. W., \& Hilz, R. (1965). Contrast sensitivity of the human eye for square-wave gratings. Journal of the Optical Society of America, 55, 1086-1091.

WILSON, R. H. (1991). Psychophysical models of spatial vision and hyperacuity. In D. Regan (Ed.), Vision and visual dysfunction: Vol. 10. Spatial vision (pp. 64-86). London: Macmillan.
Yager, D., Kramer, P., Shaw, M., \& Graham, N. (1984). Detection and identification of spatial frequency: Models and data. Vision Research, 24, 1021-1035.

\section{NOTES}

1. Our description of the predictions for the single-band and the multiple-band models is rather intuitive and lacking in some details for the sake of space and general readability. For example, our description of the multiple-band model does not refer to the probability of an irrelevant hit which occurs when one of the samples from the nonstimulated channels responding to the signal interval happens to be larger than the sample from the stimulated channel. For more rigorous mathematical treatments of the predictions, see Hübner (1993a, 1993b).

2 . The actual slope of the logistic function is $\alpha / 8$, not $\alpha$. The derivative of Equation 2 is given by

$$
P C^{\prime}(S L)=0.5 \alpha \exp [-\alpha(S L-\beta)] /\{1+\exp [-\alpha(S L-\beta)]\}^{2},
$$

so that at the threshold level $(S L=\beta), P C^{\prime}(\beta)=\alpha / 8$. In the main text, the statistical analyses and the discussion were based on the values of $\alpha$. Using $\alpha / 8$ does not change any of the statistical results or the conclusion in the text.

(Manuscript received September 13, 2000;

revision accepted for publication July 6, 2001.) 